vitamin $\mathrm{D} /$ calcium in $27 \%(29 / 109)$, furosemide in $26 \%(28 /$ $109)$, glucocorticoids in $22 \%(24 / 109)$, bisphosphonates in $7.6 \% \%(9 / 119)$, and calcitonin in 3.7\% (4/109).

Outcome information was provided in $106 / 119$ cases. $87 \%$ (92/106) reported a full resolution. Persistent calcinosis was present in $5.7 \%(6 / 106)$.

Conclusion Babies treated with therapeutic hypothermia should be closely monitored for SFNN, and development of hypercalcaemia.

\section{GP263 OCCURRENCE OF CLINICAL FEATURES AND RISK FACTORS IN CULTURE POSITIVE EARLY ONSET SEPSIS COMPARED TO NO SEPSIS IN NEONATES $\geq 35$ WEEKS GESTATION}

\begin{abstract}
${ }^{1}$ Arie Fisher*, ${ }^{1}$ Anne O'Sullivan, ${ }^{2}$ Eyal Fisher, 'Anne Doolan. 'Department of Neonatology, The Coombe Women and Infants University Hospital, Dublin, Ireland; ${ }^{2}$ Department of Applied Mathematics and Theoretical Physics, Centre for Mathematical Sciences, University of Cambridge, Cambridge, UK
\end{abstract}

\subsection{6/archdischild-2019-epa.322}

Background Despite advances in prevention strategies the diagnosis of neonatal sepsis and clinical decision making remains challenging. Empirical antibiotic treatment is given to neonates when sepsis is suspected. However, clinical and laboratory signs are generally unspecific and most neonates who receive antibiotics are not ultimately diagnosed with sepsis. Many physicians view empirical antibiotics as the safest course of action in cases of equivocal clinical presentation. The long term effects of early gut flora modification are poorly understood but some scientists suggested this may alter activation of genes involved in modulating immune responses. This study aims to provide some insight into the level of risk associated with typical indications for neonatal septic workup at our institution.

Methods We conducted a retrospective case-control study. Infants born at $\geq 35$ weeks gestation who received empirical antibiotics over a three months period were included along with all infants who were recorded to have culture positive sepsis in a ten year period. Three outcome groups were defined: (1) Culture positive sepsis $(\mathrm{N}=43)$ (2) Suspected culture negative sepsis $(\mathrm{N}=5)$ and (3) No sepsis $(\mathrm{N}=97)$. Rates of clinical symptoms and exposure to maternal and neonatal risk factors were compared. P-values were calculated using a test for equality of proportions implemented in the $\mathrm{R}$ programming language.

Results There was a statistically significant increase in red flag clinical features (mechanical ventilation, seizures, respiratory distress starting more than 4 hours after delivery and signs of shock) in the culture positive sepsis group compared to the no sepsis group $(\mathrm{p}<0.01)$. There was no statistically significant difference in the occurrence of exposure to suspected chorioamnionitis, PROM or late prematurity.

Conclusion Respiratory distress and suspected chorioamnionitis were the most common indications for a sepsis workup but neither was significantly more common in the culture positive sepsis group. Observation and repeated evaluation may be suitable for infants with equivocal presentation. Critically ill infants with red flag clinical features and infants with a greater number of clinical symptoms should have a blood culture taken and IV antibiotics commenced without delay.

\section{GP264 PROTEIN DOTATION NECESSITY IN LATE PRETERM INFANTS WITH IUGR: NEED TO REVISE GUIDELINES}

1,2Irina Belyaeva*, '2eyla Namazova-Baranova, 'Elena Bombardirova, ${ }^{1}$ Potekhina Tatiana, ${ }^{1}$ Illarionova Maria, 'Turti Tatiana. ${ }^{1}$ FSAl «National Medical Research Center for Children's Health» of the Russian Federation Ministry of Health, Moscow, Russian Federation; ${ }^{2}$ Pirogov Russian National Research Medical University, Moscow, Russian Federation

\subsection{6/archdischild-2019-epa.323}

Due to frequent perinatal pathologies, including ones that are connected with enteral nutrition intolerance, premature infants often have growth retardation that may lead to negative effects of accelerated growth and therefore to metabolic disorders in adult life.

Aim to assess dynamics of anthropometric measurements and body composition in late preterm infants (gestational age $(\mathrm{GA}) \geq 34$ weeks) with and without intrauterine growth restriction (IUGR) at the first year of life.

Materials and methods food ration, length and body weight figures, and body composition of preterm infants with GA $\geq$ 34 weeks born with and without IUGR were studied from the moment of birth till the terms corrected age, at $3^{\text {rd }}, 6^{\text {th }}$, and $12^{\text {th }}$ months corrected age. 60 preterm infants without IUGR and 42 preterm infants with IUGR were included in the study. Perinatal period characteristics in both groups of premature infants had no significant differences. Statistically significant differences based on GA were not found. Birth weight was lower in the premature infants with IUGR. Infants who was born with weight $\geq 1800 \mathrm{~g}$ received unenriched human milk or nutrient-enriched formula. Body composition was estimated by the air plethysmography method with the use of apparatus by PEA POD, Cosmed, Italy.

Results The premature infants with IUGR and GA $\geq 34$ weeks at the term corrected age had delay of growth development rates compared to the premature infants without IUGR, we associate that with the fact $87 \%$ of the infants received unenriched human milk. At the $3^{\text {rd }}$ month corrected age these infants had a 'catch-up growth' accompanied by the increase of fat mass in the body composition. At the $12^{\text {th }}$ month corrected age late preterm infants with IUGR caught up with the growth rate of preterm infants without IUGR that led to disappear of studied parameters significant differences between both groups.

Conclusion Therefore, nutrition approaches for preterm infants without IUGR are not appropriate for late preterm infants with IUGR and body weight at birth $\geq 1800 \mathrm{~g}$. These infants need increased protein dotation during the neonatal period in order to provide adequate postnatal growth and minimise postponed metabolic disorders risks.

\section{GP265 WHERE IS THE TIP? AN AUDIT ON PICC LINE INSERTION IN A TERTIARY LEVEL NEONATAL UNIT}

Tayyaba Yasmeen*, Andrew Powls. Princess Royal Maternity Hospital, Glasgow, UK

\subsection{6/archdischild-2019-epa.324}

Objectives To determine whether the Peripherally Inserted Central Catheter(PICC) line tips are at appropriate positions when inserted and used in neonates

To determine the common complications associated with PICC lines. 
Method A retrospective review of all the neonates who had PICC lines inserted from Jan2016- Jun2018 was collected from online patient data base and case notes. The online Picture Archiving and Communication System (PACS) was utilized to review all the X-rays done post procedure.

Variables including gestational age, birth weight, site of line insertion, line tip position on X-ray and its documentation in notes and formal radiology reports, any deliberate readjustment or change in line position in subsequent X-rays, duration of use, associated complications and reasons of removal were reviewed. Detailed review of the babies who suffered complications secondary to PICC line insertion was also done.

Results Total 62 PICC lines inserted in 43 babies were reviewed. The median gestational age was 27 weeks while the birth weight was $920 \mathrm{~g}$. Right arm was the most common site of insertion $(n=19,31 \%)$. The median age at line insertion was $8 d$ with $43 \%(n=27)$ lines passed during the first week of life. Median duration of use of these lines was 5 d. Only about $26 \%(n=17)$ were optimally placed in the midline veins. Subclavian vein was the most common suboptimal position for the upper limb lines $(61 \%, n=22)$ while for the lower limb was near the hip joint $(n=7,23 \%)$ or the left side of spine with potential risk of involvement of ascending lumber $\operatorname{vein}(n=5,22 \%)$. About $29 \% \quad(n=18)$ of the lines were readjusted, amongst which the line tip within the cardiac silhouette being the most common cause. None of the lines with tips potentially in the ascending lumber vein were readjusted or further investigated. The most common complication leading to early removal was line occlusion $(\mathrm{n}=8,13 \%)$.

Discussion Malposition of the PICC tips is common and may result in significant morbidity if not recognized. Recognition of malposition, other than intracardiac, is low, and additional education is required. Additional imaging is required where tip position is unclear.

\section{GP266 A RETROSPECTIVE AUDIT OF THE INCIDENCE OF HYPOGLYCAEMIA IN AT RISK NEONATES AND COMPLIANCE WITH LOCAL GUIDELINES}

Susan Keogh*, David Corcoran. Rotunda Hospital, Dublin, Ireland

\subsection{6/archdischild-2019-epa.325}

Introduction Neonatal hypoglycaemia is a common neonatal problem and there is active debate surrounding screening, associated factors and long term consequences. There is controversy surrounding the outcome of transient neonatal hypoglycaemia but Kaiser et al reported decreased proficiency in literacy and mathematics tests in these infants at follow-up, even when adjusted for gestational age. In 2011, the American Academy of Paediatrics revised the neonatal hypoglycaemia guideline, this outlined the infants at risk for hypoglycaemia i. e large for gestational age, small for gestational age, IUGR, late preterm and maternal gestational diabetes. We have adopted a similar national and local guideline for screening and management of neonatal hypoglycaemia in Ireland.

Methods We evaluated the presence of hypoglycaemia $<3$ $\mathrm{mmol} / \mathrm{l}$ in our neonatal population over a three month period. We did this using the results from the point of care blood glucose machine. We then performed a retrospective review using the NM-CMS system to view patient records for the neonate and their mother. We were able to determine whether there were any neonatal or maternal factors that caused increased risk of hypoglycaemia.

Results We were able to retrieve data on 86 episodes of hypoglycaemia in this time period. Which occurred in 55 neonates. Compliance with guideline Only $6(10.9 \%)$ had a serum glucose sent to the lab, 49 (89\%) had three pre feed glucose measurements taken prior to discharge. 23 (41.8\%) were appropriately managed with glucogel.

Risk factors We identified $8(14.5 \%)$ of infants that were late preterm, 9 (17.6\%) infants were small for gestational age, 6 $(10.9 \%)$ large for gestational age, 25(45\%) maternal diabetes, $18(32 \%)$ increased maternal BMI, 7 (12.7\%) maternal beta blocker use.

$43(78 \%)$ of the infants identified were exclusively breastfeeding, $8(14.5 \%)$ were exclusively formula feeding. and the remaining $4(7.2 \%)$ were combination feeding.40 (72.7\%) mothers were over 35 years with $34.5 \%$ over 40 years.

Conclusion This audit confirms that hypoglycaemia is prevalent in our population, however it was not possible to identify all the hypoglycaemia episodes due to inaccurate recording on the care blood glucose machine so we have likely under estimated the true prevalence. Two of the most significant risk factors we have identified are advanced maternal age and exclusive breast-feeding. This may indicate a high-risk cohort which should be included in the early blood glucose monitoring and early feeding support to prevent negative sequelae.

\section{GP267 PREVENTION OF GROUP B STREPTOCOCCUS INFECTION- RESULTS FROM A SCREENING PROGRAM}

Catarina Ferraz Liz*, Sara Soares, Abílio Oliveira.

10.1136/archdischild-2019-epa.326

Centro Hospitalar Tâmega e Sousa, Penafiel, Portugal

Introduction Group B Streptococcus (GBS) remains one of the leading causes of early and late onset sepsis in neonate. This bacteria is present in the gastrointestinal and urinary tract of pregnant woman which could lead to vertical transmission to the newborn.

Following the first statements of the American College of Obstetricians and Gynecologists and the American Academy of Pediatrics, the Center of Disease Control and Prevention (CDC) released the first guidelines in 1996 which were reviewed in 2002 and 2010.

In the last revision, CDC recommends universal screening of pregnant women between 35-37 weeks of gestation and intrapartum antibiotic prophylaxis for those with a positive screening. This recommendation led to a decrease in GBS early onset sepsis worldwide.

In Portugal, screening is a universal practice, performed in the last prenatal consultation.

The goal of this study was to verify the main outcomes of the GBS screening program in a Portuguese maternity.

Methods A retrospective study, including all newborns $>36$ weeks of gestation born between November 2015 and May 2016 in a Portuguese maternity was conducted. Data were collected from clinical records.

Results GBS screening was performed in 1090 mothers $(96,9 \%)$, with a positive rate of $22,7 \%(n=247)$.

In 74 cases there was no/inadequate intrapartum prophylaxis $(28,6 \%)$ 